\title{
Motor Axons Preferentially Reinnervate Motor Pathways
}

\author{
Thomas M. E. Brushart \\ The Raymond M. Curtis Hand Center and Departments of Orthopaedics and Neurology, Johns Hopkins University, \\ Baltimore, Maryland 21205
}

\begin{abstract}
Motor axons regenerating after transection of mixed nerve preferentially reinnervate distal motor branches and/or muscle, a process termed "preferential motor reinnervation." Collaterals of a single motor axon often enter both sensory and motor Schwann cell tubes of the distal stump; specificity is generated by pruning collaterals from sensory pathways while maintaining those in motor pathways. Previous experiments in the rat femoral nerve model evaluated reinnervation of the femoral motor branch and quadriceps muscle as a unit. In this study, pathway contributions are analyzed separately by denying muscle contact, or by reinnervating muscle through inappropriate, formerly sensory pathways. Motor axons preferentially reinnervate motor pathways, even when these pathways end blindly in a silicon tube. If the femoral nerve is removed as a graft and reinserted with correct or reversed alignment of the sensory and motor branches, more motoneurons reinnervate muscle through correct motor than through incorrect sensory pathways. Motor pathways thus differ from sensory pathways in ways that survive Wallerian degeneration and transplantation as a graft, and that can be used by regenerating motor axons as a basis for collateral pruning and specificity generation.
\end{abstract}

[Key words: specificity, pruning, nerve graft, L2/HNK-1, contact recognition, femoral nervel

Surgical "repair" of transected peripheral nerve mercly facilitates a complex reinnervation sequence. The inadequacy of the "repair" concept is frequently emphasized by the poor sensory and motor function that results. Regeneration of axons into inappropriate distal pathways is a possible mechanism for this failure (Langley and Hashimoto, 1917). Motor axons enter sensory Schwann cell tubes and are directed to skin, while sensory axons enter motor Schwann cell tubes and are directed to muscle. These misdirected axons often fail to establish functional connections, and might exclude appropriate axons from the pathways they occupy. Factors leading to appropriate sensory and motor reinnervation could reduce the resulting functional compromise.

Received Sept. 25, 1992; revised Dec. 21, 1992; accepted Jan. 29, 1993.

This work was supported by an Institutional Research Grant from Johns Hopkins University, by the Raymond M. Curtis Research Fund, and by NIH Grant P0122849. I thank Mr. Philip Kessens for superb technical assistance, Dr. Alan Kimball for statistical analyses, Ms. Joyce Lavery for artwork, and Dr. John Griffin for review of the manuscript. I am particularly grateful to the many researchers I visited as Bunnell Fellow of the American Society for Surgery of the Iland for their thoughtful discussions.

Correspondence should be addressed to Dr. Brushart, The Raymond M. Curtis Hand Center, 1400 Front Avenue, Lutherville, MD 21093.

Copyright (C) 1993 Society for Neuroscience 0270-6474/93/132730-09\$05.00/0
The search for evidence of sensory/motor specificity has produced conflicting results. Early studies of muscle spindle reinnervation (Tello, quoted in Ramon y Cajal, 1928) and crossunion of sensory and motor nerves (Langley and Anderson, 1904) showed that some motor axons escape from inappropriate sensory pathways to reinnervate their original targets. Ramon y Cajal attributed this behavior to "a neurotropic influence which has an individual and specific character" for each type of end organ (Ramon y Cajal, 1928). Enthusiasm for sensory/motor specificity was later diminished by the results of other misrouting experiments. Motor axons were found to extend over long distances through sensory pathways, and to remain stable in these foreign environments for over a year (Kilvington, 1941). Misdirected sensory axons formed persistent arborizations within muscle, but failed to support it trophically (Weiss and Edds, 1945; Zalewski, 1970). These experiments helped clarify the interaction of axons and foreign end organs. However, the axons were forcibly redirected; their behavior when confronted with equal access to distal sensory and motor environments was not tested.

A new regeneration model has been designed in response to these shortcomings (Brushart, 1988). The rat femoral nerve was chosen because of its ideal anatomical characteristics. Proximally, at the site of experimental nerve transection, sensory and motor axons intermingle throughout the nerve. As these axons regenerate, they have equal access to neighboring motor and sensory Schwann cell tubes in the distal nerve stump. This assures an element of "choice" at the axonal level not present in carlier experiments. Distally, where the specificity of regeneration is assessed, axons are segregated into terminal sensory and motor branches. These branches are well matched as competing "targets" for regenerating axons; the sensory branch contains more, smaller myelinated axons, and the motor branch fewer, larger myelinated axons. Motor axons are normally found only in the motor branch, so any motor reinnervation of the sensory branch represents a failure of specificity. In initial experiments (Brushart, 1988), HRP was used to quantify sensory and motor neurons reinnervating the sensory or motor branch. Motoneurons preferentially reinnervated the motor branch in both juvenile and adult rats, even if the repair was intentionally misaligned or a gap was imposed between proximal and distal stumps. A specific interaction thus occurred between regenerating motor axons and the motor branch and/or muscle, termed "preferential motor reinnervation" (PMR). The specificity of sensory reinnervation was more difficult to evaluate with the femoral nerve model, so further evaluation was confined to the motor system.

The original model was modified to investigate the mechanism of PMR (Brushart, 1990). A proximal $0.5 \mathrm{~mm}$ gap served 


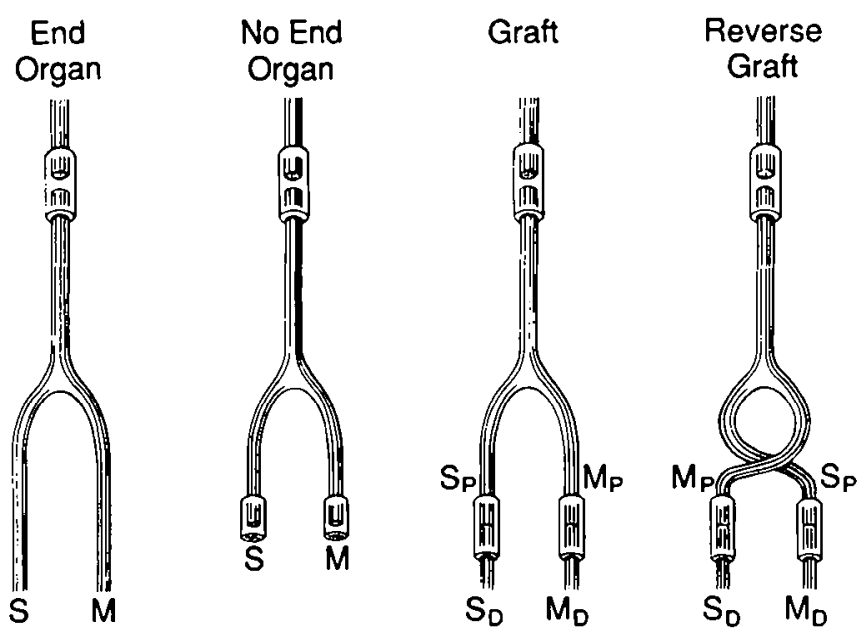

Figure 1. Manipulations of the rat femoral nerve employed in these experiments. In all groups, the proximal femoral nerve is severed and the stumps are held $0.5 \mathrm{~mm}$ apart within a silicon tube (test lesion). Treatment of the motor and sensory branches differs by group. End Organ. Motor and sensory branches left undisturbed. No End Organ, Motor and sensory branches transected at the level of muscle innervation, doubly ligated, and sewn within blind silicon tubes to prevent end-organ reinnervation. Graft. Femoral nerve removed as a graft before performing proximal juncture. Sensory and motor branches are correctly realigned within silicon tubes to maximize distal stump reinnervation while preventing shunting of axons between repairs. Muscle is reinnervated through the motor pathway (Mp to Md). Reverse Graft, Femoral nerve is again removed as a graft. However, distal stumps are incorrectly realigned, with motor leading to sensory ( $\mathrm{Mp}$ to $\mathrm{Sd}$ ), and sensory to motor (Sp to Md).

as the test lesion. This unstructured environment between nerve stumps eliminated the possibility of specificity generation through axonal alignment. Juvenile animals were used because they most strongly demonstrated PMR in initial experiments. Two, three, or eight weeks after nerve transection the specificity of motor axon regeneration was evaluated by simultaneous application of HRP to one distal femoral branch and fluoro-gold (FG) to the other. Motor axon regeneration was random at two weeks, but the number of correct projections increased dramatically at later times. Many neurons initially contained both tracers, and thus projected collaterals to both sensory and motor branches. The number of these double-labeled neurons decreased with time. Motor axon collaterals were thus pruned from the sensory branch, increasing the number of correct projections at the expense of double-labeled ncurons. However, motor axons projecting exclusively to the sensory branch were unable to correct their error, and their numbers remained constant.

The contributions of motor pathway and end organ to specificity generation are evaluated as a unit when neuromuscular continuity is preserved. The present experiments were designed to isolate pathway contributions, and to assess their interaction with those of the end organ. The femoral nerve model was further modified to prevent end-organ contact, or to allow endorgan reinnervation through an incorrect pathway (Fig. 1). PMR occurred in response to the femoral motor branch alone, demonstrating that motor axons interact specifically with old motor pathways (Brushart, 1989). This interaction was confirmed by pathway switching experiments, in which more motoneurons reinnervated muscle through correct motor than through incorrect sensory pathways. Mammalian Schwann cell tubes thus maintain a specific identity that can be recognized by regenerating axons, and that influences their subsequent behavior.

\section{Materials and Methods}

Surgical procedures. Experiments were performed on the femoral nerves of juvenile ( 3 weeks, $50 \mathrm{gm}$ ) female Sprague-Dawley rats under Chloropent anesthesia (Dodge Laboratories; $3 \mathrm{ml} / \mathrm{kg}$ ). A uniform test lesion was made bilaterally in the proximal femoral nerves of all experimental groups (Fig. 1). Each nerve was sharply severed under 20-40x magnification, and the proximal and distal stumps were sutured within a silicon tube (Dow Corning, 0.025 inch i.d.) to maintain a $0.5 \mathrm{~mm}$ gap between their surfaces. This initially unstructured gap serves to minimize the effects of intraneural anatomy on the specificity of regeneration (Brushart, 1988). Experimental animals were divided into four groups based on the initial handling of terminal motor and sensory branches (Fig. 1). In the end-organ (EO) group, the terminal branches were left undisturbed, maintaining normal contact with their appropriate sensory and motor end organs. In the no end-organ (NEO) group, the motor branch was transected distally at the level of muscle innervation, and the sensory branch at an equal distance from the femoral nerve bifurcation. The proximal stumps were doubly ligated and sewn within blindended silicon tubes to deny end-organ contact to regenerating axons. In graft $(G)$ and reverse-graft (RG) groups, the femoral nerve was removed as a graft. All proximal junctures were performed as described above, and distal junctures were end to end within supporting silicon tubes to maximize distal stump reinnervation. In group $G$ the distal stumps were aligned correctly [proximal sensory (Sp) to distal sensory (Sd) and proximal motor $(\mathrm{Mp})$ to distal motor (Md)], and in group $\mathrm{RG}$ they were crossed (Mp to Sd, Sp to Md). Md consisted of the terminal $2 \mathrm{~mm}$ of femoral motor branch as it entered the quadriceps muscle. Sd extended a minimum of $10 \mathrm{~mm}$ to local skin, with saphenous fascicles reaching the foot. Eight groups of 15 rats each were prepared; regeneration was assessed at 2, 3, and 8 weeks in groups EO-2, EO-3, and EO-8, and at similar time intervals in groups NEO-2, NEO-3, and NEO-8. All animals in groups $G$ and $R G$ survived for 8 weeks. Proximal tubes were removed from all 8 week animals after 2 weeks of regeneration to prevent late compression of the repairs. Animals with a change in the interstump gap, as shown by suture migration, were excluded from the study at that time. The distal tubes in NEO groups remained in place throughout the experiments; those in groups $G$ and $R G$ were removed at 2 weeks and a nonconstricting sheet of Saran Wrap was placed between junctures to prevent escape of axons to the opposite pathway.

Perfusion and histochemistry. At the end of the regeneration interval, the femoral sensory and motor branches were exposed bilaterally. The motor branch was severed as it entered the quadriceps muscle in all EO groups and in groups $\mathrm{G}$ and RG (through Md), and within the blocked tube in NEO groups. The sensory branch was cut an equivalent distance from the femoral nerve bifurcation to produce proximal sensory and motor stumps of equal length. One stump (randomly chosen) was exposed to $10 \%$ HRP (Sigma VI) for $1 \mathrm{hr}$ in a Vaseline well, after which it was copiously irrigated and loosely sutured to a distant portion of the wound. The other stump was then exposed to $3 \%$ fluoro-gold (FG) (Schmued and Fallon, 1986; Brushart, 1990) for $2 \mathrm{hr}$, similarly irrigated, and sewn to the opposite corner of the wound to prevent cross-contamination by diffusion of tracers. Forty-eight hours were allowed for proximal transport of the tracers, after which the animals were deeply anesthetized and perfused through the left ventricle. A warm saline flush $(150 \mathrm{ml})$ was followed by $500 \mathrm{ml}$ of $4 \%$ paraformaldehyde in $0.1 \mathrm{M}$ sodium acetate buffer (pH 6.5) over $0.5 \mathrm{hr}, 500 \mathrm{ml}$ of $4 \%$ paraformaldehyde in 0.1 m sodium borate buffer $(\mathrm{pH} 9.5)$ over $0.5 \mathrm{hr}$, and $500 \mathrm{ml}$ of $10 \%$ sucrose in $0.1 \mathrm{~m}$ Sorensen's phosphate buffer $(\mathrm{pH} 7.4)$ over 0.5 hr (Berod et al., 1981; Mesulam, 1982). The proximal tube, previously undisturbed in groups EO-2, EO-3, NEO-2, and NEO-3, was examined after perfusion; animals in which suture migration suggested a change in interstump gap were excluded. The lumbar spinal cords of remaining animals were removed and serial cross sections were cut at $40 \mu \mathrm{m}$ on a freczing microtome. These sections were reacted with $\mathrm{H}_{2} \mathrm{O}_{2}$ and tetramethylbenzidine to demonstrate HRP within motoneurons (Mesulam, 1982). Sections were serially mounted on glass slides, dried, and coverslipped without counterstain to minimize background fluorescence.

Evaluation of results. Each section was viewed with fluorescent (405 $\mathrm{nm}$ ) and transmitted light at $20-40 \times$ by an observer unaware of which tracer had been used on which femoral branch. Counts were prepared for each nerve of (1) HRP-labeled motoneurons, (2) FG-labeled motoneurons, and (3) double-labeled motoneurons. The presence of split cells in adjacent sections was corrected for by the method of Abercrombie (1946). Data were compiled for the first 10 animals (20 nerves) to complete the study successfully within each of the eight groups (total 
Figure 2. Motoneuron counts from EO and NEO groups at 2,3, and 8 weeks. Each triad of vertical bars represents the mean counts obtained from exposure of 20 nerves to HRP/FG. Motoneurons are scored as projecting correctly to the motor branch (white bar), incorrectly to the sensory branch (black bar), or simultaneously to both branches (double labeled; stippled bar). At 2 weeks regeneration is random in both $\mathrm{EO}$ and NEO groups. By 3 weeks the number of correct projections to the motor branch increases markedly, while the number of incorrect projections to the sensory branch remains relatively constant. The sensory/motor difference becomes even more prominent at 8 weeks; it is consistently more pronounced at both 3 and 8 weeks in the EO group. The number of double-labeled neurons, those with collateral projections to both branches, decreases significantly at each time period in both EO and NEO from the sensory branch, increasing the number of correct projections at the expense of double-labeled neurons. groups. Axon collaterals are thus pruned

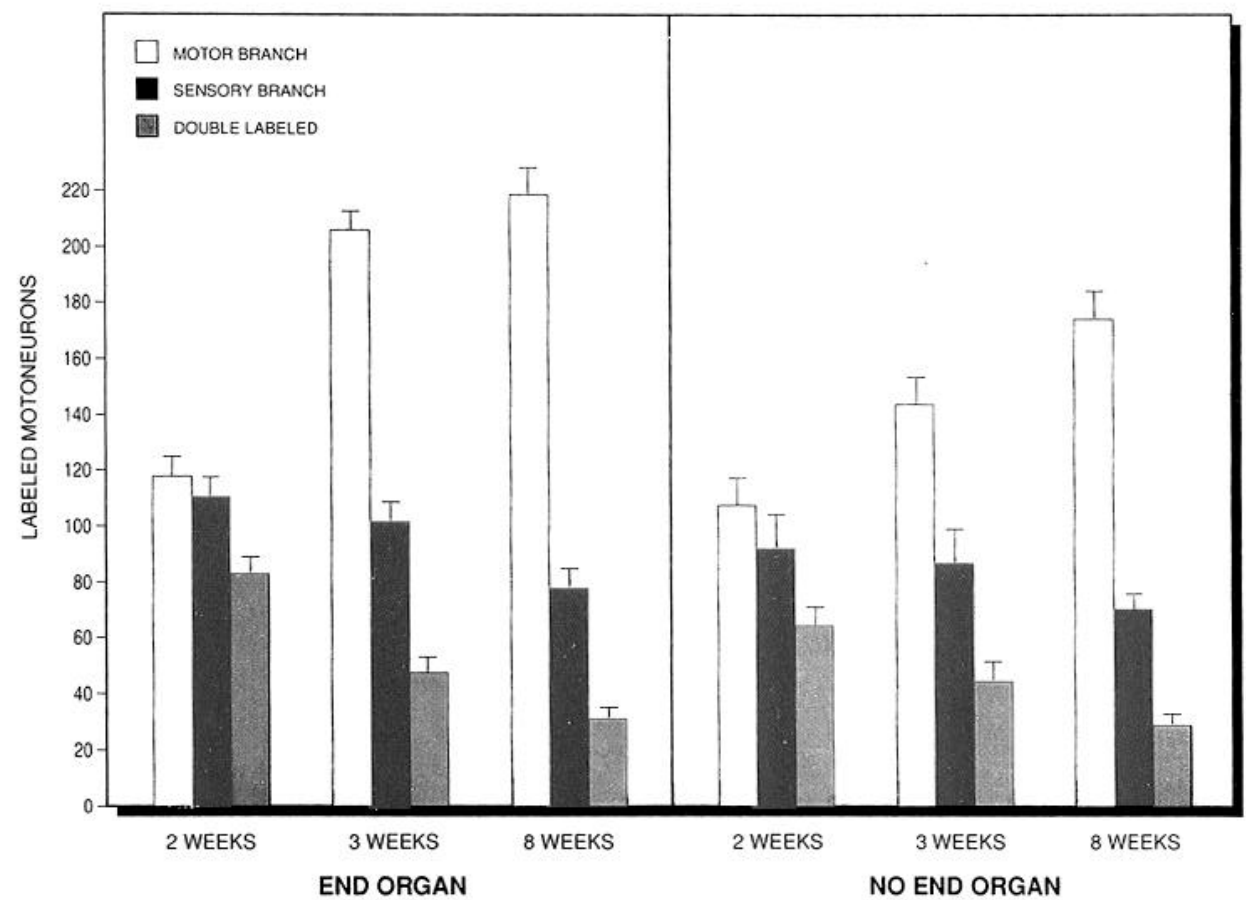

160 nerves). Each group was then characterized by three means: the mean number of motoneurons projecting correctly to the motor branch (Md in $\mathrm{G}$ and $\mathrm{RG}$ groups), the mean number projecting incorrectly to the sensory branch (Sd in G and RG groups), and the mean number of double-labeled neurons, those projecting axon collaterals to both branches. Paired $t$ test analyses compared correct, incorrect, and doublelabeled neuron counts within each of the eight groups; unpaired $t$ tests were used to compare the motor/sensory difference (the difference between the mean of correct motor and the mean of incorrect sensory projections) among groups EO-2, EO-3, and EO-8, among groups NEO2 , NEO-3, and NEO-8, and between groups $\mathrm{G}$ and RG. In addition, Duncan's multiple range test was applied to the means generated by a $3 \times 3 \times 2$ factorial analysis of variance (three time periods, three types of labeling, presence or absence of end organ) to evaluate further the relative contributions of pathway and end organ to specificity across all EO and NEO groups.

Control experiments. The anatomical characteristics of the femoral nerve model have been described previously (Brushart, 1988). Control experiments have demonstrated that HRP and FG may be reliably confined to the intended pathway in this model, and resulting motoneuron counts in normal animals are comparable with the two techniques (Brushart, 1990). As a further precaution, the choice of tracer is made randomly for each branch to defeat any preferential uptake or transport of one tracer that might be a unique property of regenerated axons. Further controls were required by the various manipulations of the terminal sensory and motor branches in the present experiments. In the animals denied end-organ contact (NEO), the permanence of each lesion was tested by stimulating the femoral nerve trunk in situ at the time of HRP/FG application. A $2 \mathrm{~mA}$ stimulus was delivered with a Vari-Stim III stimulator (Concept, Inc.) and the quadriceps muscle was examined for contraction. Nerve stimulation with muscle recording was not practical, as the dissection and manipulation required to isolate the nerve electrically on a stimulating electrode were found to interfere with axoplasmic transport and labeling, probably through transection of nutrient vessels. In graft $(G)$ and reverse-graft $(R G)$ animals, regenerating axons must not escape from the distal junctures to reinnervate the opposite, unintended pathway. Ten nerves underwent crossed repair of sensory and motor branches as in group RG, but with no proximal lesion. In this preparation motor axons could only reach the distal motor branch by escaping from the $\mathrm{Mp}$-to-Sd repair and crossing over to enter the Sp-to-Md repair. The success of repair isolation was assessed after 8 weeks of regeneration by applying HRP to the distal motor branch and examining the spinal cord for inappropriately labeled motoneurons. In evaluating the interaction of pathway and end organ, it is also essential to know the precise timing of end-organ reinnervation. An additional
20 nerves were prepared as in group EO to assess the timing of motor end-plate reinnervation. Four quadriceps muscles each were harvested at $8,11,14,17$, and $20 \mathrm{~d}$, frozen in isopentane, and cut at $40 \mu \mathrm{m}$ with a cryostat. A combined silver-cholinesterase stain clearly demonstrated the relationship between regenerating axons and neuromuscular junctions at each time period (Pestronk and Drachman, 1978) (see Fig. 4).

\section{Results}

\section{End organ, no end organ}

The EO experiments have been described previously (Brushart, 1990) and are presented here as controls for the NEO groups (Fig. 2). Comparison of NEO and EO results shows the time course of specificity generation to be similar with and without end organ. However, more motoneurons are labeled from the motor branch when muscle reinnervation is permitted. In group NEO-2 the mean numbers of correctly and incorrectly projecting motoneurons do not differ significantly [motor branch projections $(M)=106$, sensory branch projections $(S)=96 ; p=0.60]$. During the next week, the mean number of correct projections to the motor branch increases markedly $(M=142)$, and the mean number of incorrect projections to the sensory branch shows a slight decline $(\mathrm{S}=82)$. This tendency is more prominent at 8 weeks $(M=175, S=70)$. Preferential reinnervation of the motor branch by motor axons thus occurs at 3 and 8 weeks ( $p$ $=0.0001)$ even when end-organ contact is denied. The mean number of double-labeled motoneurons (DL) decreases significantly at each interval (DL $=62$ at 2 weeks, 43 at 3 weeks, and 26 at 8 weeks; $p=0.026$ for these intervals). Further analysis was performed to see if correct reinnervation was more likely in the presence or absence of the end organ. Duncan's multiple range test was applied to the means generated by a $3 \times 3 \times 2$ factorial ANOVA at the $p=0.05$ level. The number of motoneurons projecting correctly down the motor branch was independent of end-organ contact at 2 weeks, but greater when the end organ was present at 3 and 8 weeks. The number of motoneurons projecting incorrectly down the sensory branch was independent of end-organ contact at all time periods. Dou- 


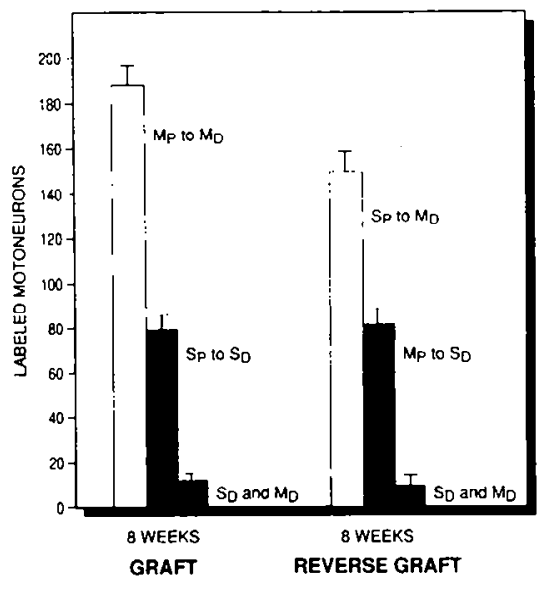

Figure 3. Motoneuron counts obtained at 8 weeks in G and RG groups. A triad of vertical bars represents the mean counts from 20 femoral nerves. Each bar is labeled both as to the pathway taken and the destination from which axons were labeled. For instance, motoneurons included in the "Mp to Md" bar have regenerated through the motor pathway $(\mathrm{Mp})$ to reinnervate muscle through the very short distal motor stump (Md), from which they were labeled. White bars represent labeling from Md (leading to muscle), black bars represent labeling from Sd (leading to skin), and stippled bars represent double labeling, those neurons that project collaterals to both $\mathrm{Sd}$ and Md. Significantly more motoneurons reinnervate muscle through the correct, motor pathway (Mp) than through the incorrect, sensory pathway (Sp). However, equal numbers of motoneurons reinnervate skin regardless of the pathway taken.

ble labeling was less at 2 weeks without the end organ, but otherwise independent of end-organ contact.

Graft, reverse graft

The $G$ and $R G$ groups were analyzed separately from the EO and NEO groups (Figs. 1, 3). The results of small pilot experiments at early time periods were variable, possibly reflecting the presence of two nerve junctures and the variable course of graft revascularization and subsequent Schwann cell survival. Each group was thus evaluated at the 8 week time period only. Tracers were applied distally to the grafts by transecting Md and Sd (Fig. 3). Reinnervation of Sd (leading to skin) by motoneurons was independent of the pathway taken (G, Sp to Sd, mean $=81 ; \mathrm{RG}, \mathrm{Mp}$ to $\mathrm{Sd}$, mean $=83$ ). Similarly, the mean number of motoneurons that continued to project collaterals to both Md and Sd (double labeled) after 8 weeks was equally small in both groups $(G, 9 ; R G, 8)$. However, when reinnervation of Md (leading to muscle) was evaluated, significantly more motoneurons were found to reinnervate $\mathrm{Md}$ through the correct motor pathway $(\mathrm{G}, \mathrm{Mp}$ to $\mathrm{Md}$, mean $=186)$ than through the incorrect sensory pathway $(\mathrm{RG}, \mathrm{Sp}$ to $\mathrm{Md}$, mean $=145 ; p=$ $0.014)$.

\section{Controls}

Control experiments to validate the femoral nerve model and the use of HRP/FG double labeling have been reported previously (Brushart, 1988, 1990). However, additional controls were performed to assess the timing of muscle reinnervation in EO groups, to assure that no muscle reinnervation occurred after NEO lesions, and to evaluate the possible spread of axons in $G$ and $\mathrm{RG}$ groups. Combined silver-cholinesterase stain of quadriceps muscle revealed the time coursc of reinnervation (Fig. 4). Axons were totally absent from muscle $8 \mathrm{~d}$ after nerve repair, and sparse at $11 \mathrm{~d}$. Axons were more plentiful at $14 \mathrm{~d}$, though motor end-plate reinnervation was infrequent. By $20 \mathrm{~d}$ reinncrvation was complete in some areas of the muscle, but scattered clusters of end plates were still without axons. In NEO groups, electrical stimulation of the femoral nerve trunk resulted in no visible contraction of the atrophied quadriceps muscle. These findings contrasted markedly with those of earlier pilot experiments in which various combinations of ligation and interruption without the silicon barrier were ineffective blocks to regeneration. Analysis of $G$ and $R G$ results is predicated on the assumption that regenerating axons remain within the pathways to which they are directed. This was tested by preparing 10 nerves with distal crossed repairs and no proximal lesion, similar to the original experiments of Weiss and Edds (1945). If successful, this maneuver would direct all motor axons into the distal sensory branch. Application of HRP to the distal motor branch labeled no motoneurons in seven preparations, and one apiece in the remaining three, confirming the success of the procedure. In eight initial trials in which the Saran Wrap barrier was not used, a mean of 14 motor axons crossed to the oppositc repair.

\section{Discussion \\ Collateral pruning}

The time course of reinnervation, both with and without end organ, strongly suggests that PMR is generated by pruning of motor axon collaterals from the sensory branch (Brushart, 1990). At 2 weeks, similar numbers of motoneurons correctly reinnervate the motor branch and incorrectly reinnervate the sensory branch. The peripheral destination of these axons is determined at the repair site, as axons are usually confined to a single Schwann cell tube throughout their distal course (Brown and Hopkins, 1981; Brown and Hardman, 1987). Motor axons labeled at 2 weeks have thus entered sensory and motor Schwann cell tubes of the distal stump on a random basis. In contrast, neurotropic, contact recognition, or mechanical guidance of motor axons to "correct" Schwann cell tubes would result in immediate specificity. By 3 wecks, the number of motoneurons projecting correctly to the motor branch in both EO-3 and NEO-3 groups increases dramatically. This change is accompanied by a significant decrease in the number of double-labeled neurons, while the population of motoneurons projecting to the sensory branch alone decreases only slightly. These trends are even more apparent at 8 weeks. To be double labeled, neurons must reinnervate both motor and sensory branches with collaterals of a single parent axon. The significant decrease in double labeling at both 3 and 8 weeks is most readily explained by pruning of the collateral or collaterals from one of these branches. During this pruning process the number of motoneurons projecting only to the sensory branch remains constant, while the number projecting only to the motor branch increases dramatically. Axon collaterals must therefore be selectively pruned from the sensory branch, creating correct motor branch projections at the expense of double-labeled neurons. As shown in NEO groups, end-organ contact is not required for this process. The importance of collateral pruning is emphasized by the fate of motoneurons projecting only to the sensory branch. Without a collateral in the motor branch they have no means of correcting their initial error, and will persist in nearly constant numbers throughout the experiment as observed by Kilvington (1941).

The pruning hypothesis just described assumes a strict onefor-one conversion of double-labeled neurons to correctly pro- 

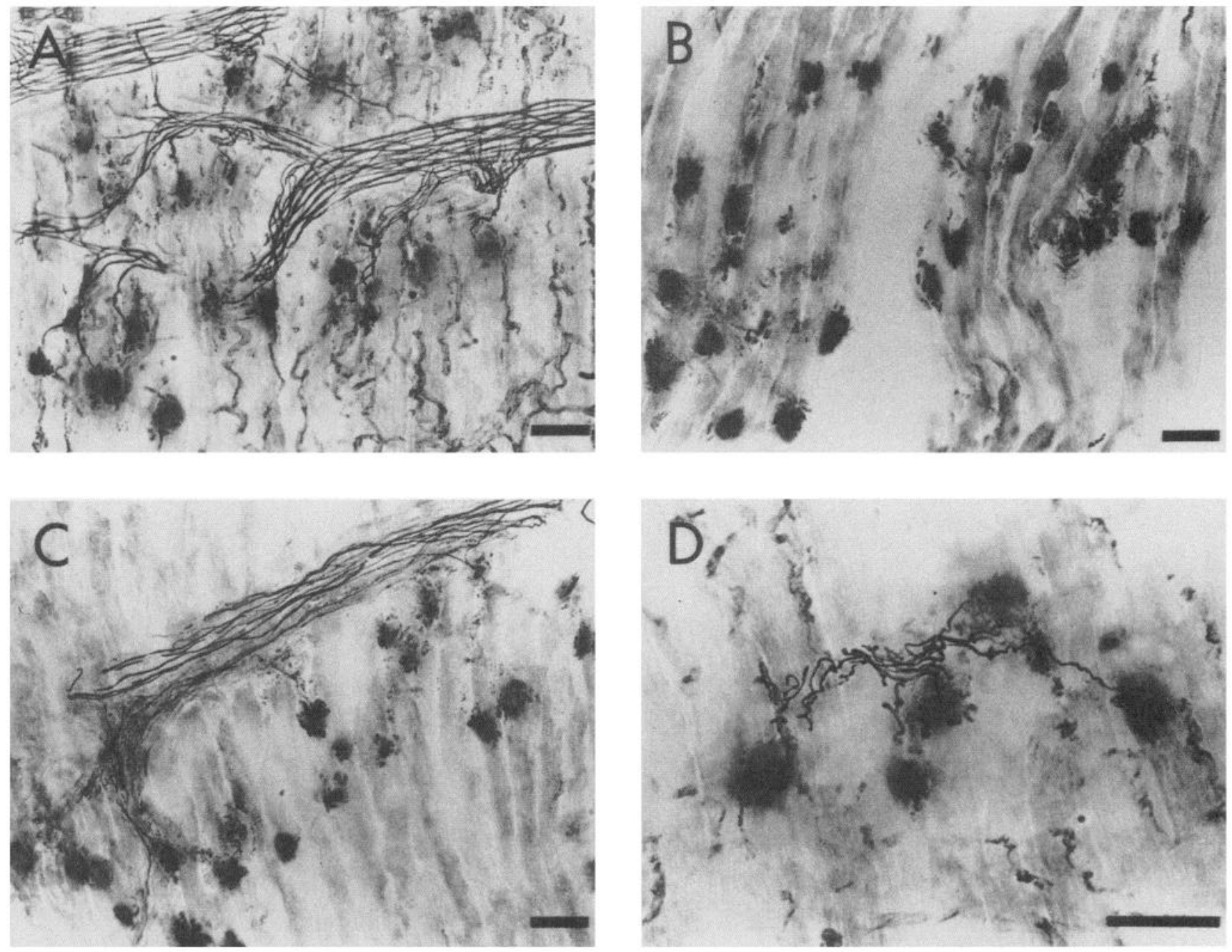

Figure 4. Rat quadriceps muscle examined with silver-cholinesterase stain. $A$, Normal muscle. $B$, Denervated muscle $8 \mathrm{~d}$ after proximal femoral repair. $C$, Muscle undergoing initial reinnervation $14 \mathrm{~d}$ after repair. $D$, Higher-power view of reinnervated end plates $20 \mathrm{~d}$ after repair. Scale bar, $50 \mu \mathrm{m}$.

jecting neurons. However, in both EO and NEO groups, at each time interval the increase in correct projections is greater than the decrease in double-labeled neurons. Most probably, the tracing studies provide "snapshots," and thus incomplete understanding, of a dynamic process. Neurons not labeled at 2 weeks may subsequently extend collaterals into both branches, and have those in the sensory branch pruned before being identified for the first time as correct projections at 3 weeks. An alternative interpretation must also be considered: swiftly regenerating motoneurons project randomly, while their slower counterparts make initially correct projections. The observed constancy of incorrect motoneuron projections to the sensory branch after 2 weeks in EO and NEO groups is consistent with a brief, early period of axon misdirection. However, this interpretation cannot encompass the repeatedly demonstrated phenomenon of collateral pruning. If pruning does not generate specificity, it must occur randomly. The inevitable consequences, a gradual increase in unbranched projections to both sensory and motor branches, are not observed (see above). The need for two entirely separate motoneuron behaviors and the failure to account for the consequences of pruning render this alternative interpretation implausible.

An abundant supply of axon collaterals is available to serve as the substrate for pruning during regeneration of mammalian nerve. Transected axons produce multiple collateral sprouts that advance distally as the "regenerating unit" (Morris et al., 1972). As a result, myelinated axon counts in the distal stump of motor nerve are elevated by a factor of 1.5-5 for several months after nerve transection and repair (Shawe, 1955; Evans and Murray, 1956; Jenq and Coggeshall, 1984). In a recent sequential study of rat sciatic nerve regeneration, Mackinnon et al. (1991) found the number of myelinated distal stump axons to be almost twice normal at 3 months, and then to diminish gradually until normal numbers were reached at 24 months. Motor axon collateral sprouts are also functionally competent. They support muscle when a small motor nerve is crossed to reinnervate a larger muscle mass (Kilvington, 1941; Thomas and Davenport, 1949), and may regenerate into separate motor nerves to reinnervate functionally two different muscles from the same neuron (Langley and Anderson, 1904; Watrous, 1940; Esslen, 1960).

Collateral pruning is a normal component of motor system development. In the neonatal limb, muscle cells are innervated by several axons (Redfern, 1970); terminal axon collaterals are then pruned to "fine tune" the motor unit (Brown and Booth, 1983). Pruning also generates specific projections during CNS development and regeneration (Innocenti, 1981; Ivy and Kil- 
lackey, 1982; Murray and Edwards, 1982; Stanficld and O'Leary, 1985; Stelzner and Strauss, 1986). The EO experiments, initially reported elsewhere (Brushart, 1990) and here used as controls for the NEO groups, were the first indication that collateral pruning could also generate specificity during vertebrate motor axon regeneration. The NEO experiments confirm and strengthen these findings. A peripheral mechanism found initially in the cockroach (Denburg, 1988) may also be available to regenerating mammalian axons. In this instance, however, it is not used to generate topographic specificity within the motor system, but to separate sensory and motor systems from each other.

\section{Pathway specificity}

The NEO experiments demonstrate a role for the peripheral motor pathway in specificity generation. The temporal pattern of motoneuron labeling without end-organ contact strongly resembles that when the end organ is present (Fig. 2). Similar numbers of motoneurons are labeled from the motor and sensory branches at the initial time period (NEO-2). The next week is characterized by an increase in the number of correctly projecting neurons; the number of double-labeled neurons drops significantly, and the number of incorrect sensory projections remains constant. These trends are accentuated at 8 wetiks. The process of specificity generation through collateral pruning thus occurs within motor pathways, without need for end-organ contact. Degenerating sensory and motor Schwann cell tubes must differ in some way that regenerating motor axons can detect, and that influences their subsequent behavior.

The $G$ and $R G$ experiments (Figs. 1, 3) provide further evidence of axon-pathway interactions. After 8 weeks of regeneration, significantly more motoneurons reinnervate Md (leading to muscle) through the "correct," former motor pathway (Mp) than through the "incorrect," former sensory pathway (Sp). Factors responsible for this difference are clarified by separation of regenerating axons into discrete populations, and comparison of the behavior of these populations in $\mathrm{G}$ and $\mathrm{RG}$ groups. When grafts are correctly aligned $(G), M d$ is reinnervated by (1) motor axons that project randomly to the motor pathway $(\mathrm{Mp})$ alone and (2) motor axon collaterals that propagate through the motor pathway $(\mathrm{Mp})$ and mature with muscle contact. When grafts are reversed (RG), Md is reinnervated by (1) motor axons that project randomly to the sensory pathway $(\mathrm{Sp}$ ) alone and (2) motor axon collaterals that propagate through the sensory pathway (Sp) and mature with muscle contact. Unbranched projections to $\mathrm{Sp}$ and $\mathrm{Mp}$ (item 1 above) are random, virtually equal, and persistent, as demonstrated by the results of EO and NEO experiments. The difference between $\mathrm{G}$ and $\mathrm{RG}$ groups must therefore reflect a difference in collateral survival (item 2 above). Any motor axon collateral that reaches muscle appears to be "rescued," presumably through trophic interactions (reviewed in Gordon et al., 1991). This "rescue" is demonstrated by the survival of twice as many motoneurons projecting through $\mathrm{Sp}$ when the eventual target is muscle (labeled from Md) than when it is skin (labeled from Sd) (Fig. 3; discussed further below). The difference between $G$ and $R G$ must therefore reflect a difference in axon collateral survival within the environments of $\mathrm{Mp}$ and Sp. Although direct evidence cannot be provided by experiments at only one time period, the findings are consistent with pruning of motor axon collaterals from the sensory pathway $(\mathrm{Sp})$, but not from the motor pathway (Mp), before muscle is reached.

Several pathway characteristics must be considered as poten- tial specificity determinants. Among the less likely are individual axon caliber and axoplasmic contents. Schwann cell tubes of the motor branch are consistently larger than those of the sensory branch (Brushart, 1988). Conceivably, they could promote survival of motor axon collaterals by allowing them to attain greater size at early time periods than they would in smaller, formerly sensory tubes. However, motor collaterals within a Schwann cell tube are initially quite small, and must compete for space with Schwann cells and other axons, a process not currently understood. Furthermore, early light microscopic studies showed that attempts to limit caliber by redirecting axons into normally small Schwann cell tubes were defeated when appropriate end-organ contact was established and caliber increased significantly (Sanders and Young, 1946). Mechanical control of PMR by the caliber of Schwann cell tubes in the distal stump thus seems unlikely. Similarly, axoplasmic contents differ between sensory and motor axons, and these differences could serve as clues to regenerating axons. Motor axoplasm contains the transmitter-specific enzymes AChE (Gruber and Zenker, 1973) and ChAT (Engel et al., 1980), while sensory axoplasm contains carbonic anhydrase (Riley et al., 1988). Even if these molecules could serve as the basis for specificity generation, the rapid pace of Wallerian degeneration in the rat (Miledi and Slater, 1970) clears them before growth cones arrive (Liu and Zhu, 1990), eliminating the possibility of their significance in this model.

PMR could reflect selective production of a "motor nerve growth factor" within the motor pathway. Possible candidates for this factor include ciliary neurotrophic factor (Manthorpe et al., 1986; Arakawa et al., 1990; Sendtner et al., 1990; Oppenheim et al., 1991), insulin-like growth factor (Hansson et al., 1986; Ishii, 1989; Caroni and Grandes, 1990), basic fibroblast growth factor (Danielson et al., 1988; McManaman et al., 1989; Grothe et al., 1991), and ChAT development factor (McManaman et al., 1988, 1989, 1991). To generate PMR, a molecular factor must reside in motor but not in sensory nerve, have a selective effect on motor neurons but not on sensory or sympathetic neurons, and survive Wallerian degeneration. No factor characterized to date satisfies these criteria.

Direct evidence for pathway recognition by regenerating peripheral axons has previously been obtained only in the leech (Peinado et al., 1987a,b). However, a pathway marker has recently been identified in mammals that satisfies the above criteria for determination of PMR. The antibodies L2 and HNK-1 identify a carbohydrate epitope (termed "L2 carbohydrate") that is associated with several neural cell adhesion molecules (Kunemund et al., 1988; Schachner, 1990). The L2 carbohydrate is selectively expressed on the Schwann cells and Schwann cell basement membrane of motor axons, but is rarely found on sensory axons (Martini et al., 1988). It persists in these locations during and after Wallerian degeneration (Brushart et al., 1992; Martini et al., 1992). The L2 carbohydrate also selectively promotes motor neurite outgrowth in vitro (Martini et al., 1992). Of particular significance is the demonstration of this selective effect on cryostat sections of the femoral sensory and motor branches. Motor neurites preferentially elongate on motor branch as opposed to sensory branch; sensory neurites obtain the same length on both substrates. L2 antibodies reduce motor neurite outgrowth on motor branch, but not on sensory branch. Sensory neurite outgrowth is not altered by $\mathrm{L} 2$ antibodies. The L2 carbohydrate thus selectively marks motor pathways, is present in the proper cellular location and at the proper time to influence 
regeneration, and has a selective effect on motoneurons in vitro. As a result, the L2 carbohydrate is currently the leading candidate for the molecular determination of PMR within peripheral pathways.

\section{Pathway-end-organ interactions}

Evaluation of reinnervation specificity with and without endorgan contact reveals not only pathway contributions, but also the interaction of pathway and end organ. Application of Duncan's multiple range test to EO and NEO groups demonstrated the number of motoneurons projecting correctly down the motor branch to be independent of end-organ contact at 2 weeks, the time at which end-plate reinnervation has just begun (Fig. 4). The number of correct projections is significantly greater in EO groups at 3 and 8 weeks, after restoration of neuromuscular continuity. Motoneuron projection to the sensory branch is independent of end-organ contact at all time periods.

The $\mathrm{G}$ and $\mathrm{RG}$ experiments also demonstrate the interaction of pathway and end organ. This is graphically illustrated in Figure 3. When the target is muscle (reached through $\mathrm{Md}$ ), pathway (Mp vs $\mathrm{Sp}$ ) has a significant effect on the number of motoneurons labeled from Md. However, when the target is skin (reached through $\mathrm{Sd}$ ), pathway has no effect on the number of motoneurons labeled from $\mathrm{Sd}$. An additional example of target effect is provided by the fate of motoneurons propagating through $\mathrm{Sp}$. Although equal numbers of motor axons presumably enter $\mathrm{Sp}$ at the repair site in both groups, almost twice as many motoneurons are labeled when muscle contact is made (Md) as are labeled when it is prevented (Sd). This difference is not likely to reflect differential uptake of tracer by active versus inactive motor axons (Kanda et al., 1989; Sato et al., 1989); preservation of motor axon collaterals through muscle contact is a more likely possibility. These findings suggest a graded motoncuron response to pathway and muscle interactions. Without either motor pathway or muscle, or with only brief pathway exposurc, specific interactions do not occur. More prolonged exposure to motor pathways results in collateral pruning and specificity generation. However, the greatest specificity is seen when both motor pathway and muscle are present.

A graded response in specificity gencration is compatible with the action of "sibling neurite bias" as the final mechanism of collateral pruning (Smalheiser and Crain, 1984). The "sibling neurite" model states that a finite pool of structural precursors is available within the neuron (Devor and Schneider, 1975). Growing neurites that consume structural components at higher rates deplete the pool available to their less vigorous "siblings." A neurite growing successfully down the motor branch, obtaining larger size because of positive interaction with the pathway (Goldberg and Schacher, 1987), would deplete the resources available to its sibling in the sensory branch, ultimately leading to resorption of this incorrect projection. A neurite receiving trophic support from muscle would mature even more rapidly than one in a correct pathway, and the pathway and end-organ effects would be additive. In this way a collateral projection can be removed from a neutral environment such as the sensory pathway without the need for a negative interaction. Similarly, when all collaterals of a motoneuron are trapped in this neutral environment, "sibling neurite bias" provides no mechanism for eliminating them all and starting over; this prediction is consistent with the stability of incorrect projections to the sensory branch throughout these experiments.

The present experiments demonstrate a role for the peripheral pathway in the generation of PMR, and suggest a mechanism by which this may occur. However, details of the experimental model must be considered before attempting to interpret these findings more broadly. All experiments were performed on 3-week-old animals because they most vigorously demonstrated PMR in previous experiments, yet have completed the period of physiologic motoneuron death (reviewed in Brushart, 1988). PMR decreases with age, and does not occur after end-to-end suture in aged rats (Brushart, 1991b). The test lesion is made at a carefully chosen level of the femoral nerve where sensory and motor axons interminglc; regenerating motor axon collaterals will thus have access to both sensory and motor Schwann cell tubes in the distal stump. This intermingling is further assured by placement of a $0.5 \mathrm{~mm}$ gap between nerve stumps. PMR has thus been demonstrated within a single nerve fascicle where sensory and motor axons are in intimate contact. Attempts to demonstrate selective reinnervation of an entire fascicle by motor axons have produced conflicting results. When regenerating motor axons are given a choice of sensory and motor fascicles within a Y-shaped silicon chamber, PMR is confirmed to varying degrees (Brushart and Seiler, 1987; Ochi et al., 1990). However, no specificity is found when proximal and distal stumps are sutured parallel to one another in the retroperitoneal environment (Maki, 1991). Details of each model must therefore be closely analyzed when attempting to reconcile disparate results. It is similarly inappropriate to generalize from the mechanism of PMR to that underlying other forms of specificity. Within both sensory and motor systems, axons have the potential for end-organ or topographic specificity (reviewed in Brushart, 1991a). So far there is no evidence linking the mechanism of PMR to generation of these other specificity types.

The results presented here suggest strategies for the enhancement of regeneration in the clinical setting. Stimulation of collateral axon sprouting may be used to enhance PMR after nerve repair. If more axons sample both sensory and motor environments, more should ultimately reinnervate the motor pathway. Tailored nerve grafts with sensory and motor branches arising from a parent trunk may be used to generate specificity during peripheral nerve reconstruction. Grafts of motor nerve should also bc compared to the standard cutaneous nerve grafts for peripheral nerve reconstruction. In this instance the issue is not sensory/motor specificity, but the propagation of motor axons over long distances in motor as compared with sensory environments.

Demonstration of specificity generation through collateral pruning may answer a long-standing question. Regenerating peripheral axons have been known to generate multiple sprouts since the time of Ramon y Cajal. The sprouting process requires significant expenditure of energy and materiel by the neuron, and must therefore be subjected to evolutionary pressure. Does the persistence of collateral sprouting imply a benefit to the organism, or is it mere evolutionary baggage, preserved because of components shared with a more crucial process? The experimental results described here suggest the former, and for the first time ascribe purpose to a long-recognized feature of peripheral axon regeneration.

\section{References}

Abercrombie M (1946) Estimation of nuclear population from microtome sections. Anat Rec 94:239-247.

Arakawa Y, Sendtner M, Thoenen H (1990) Survival effect of ciliary neurotrophic factor (CNTF) on chick embryonic motoneurons in cul- 
ture: comparison with other neurotrophic factors and cytokines. J Neurosci 10:3507-3515.

Berod A, Hartman BK, Pujol JF (1981) Importance of fixation in immunohistochemistry: use of formaldehyde solutions at variable $\mathrm{pH}$ for the localization of tyrosine hydroxylase. J Histochem Cytochem 29:844-850.

Brown MC, Booth CM (1983) Postnatal development of the adult pattern of motor axon distribution in rat muscle. Nature 304:741742.

Brown MC, Hardman V (1987) A reassessment of the accuracy of reinnervation by motoneurons following crushing or freezing of the sciatic or lumbar spinal nerves of rats. Brain 110:695-705.

Brown MC, Hopkins WG (1981) Role of degenerating axon pathways in regeneration of mouse soleus motor axons. J Physiol (Lond) 318 365-373.

Brushart TM (1988) Preferential reinnervation of motor nerves by regenerating motor axons. J Neurosci 8:1026-1031.

Brushart TM (1989) Preferential motor reinnervation: pathway regulation. Soc Neurosci Abstr 15:333.

Brushart TM (1990) Preferential motor reinnervation: a sequential double-labeling study. Restor Neurol Neurosci 1:281-287.

Brushart TM (1991a) The mechanical and humoral control of specificity in nerve repair. In: Operative nerve repair and reconstruction (Gelberman RH, ed), pp 215-230. Philadelphia: Lippincott.

Brushart TM (1991b) The specificity of motor axon regeneration decreases with age. Soc Neurosci Abstr 17:1481.

Brushart TM, Seiler WA (1987) Selective reinnervation of distal motor stumps by peripheral motor axons. Exp Neurol 97:289-300.

Brushart TM, Martini R, Schachner M (1992) Expression of L2/HNK-1 in reinnervated peripheral nerve. Soc Neurosci Abstr 18:1462.

Caroni P, Grandes P (1990) Nerve sprouting in innervated adult skeletal muscle induced by exposure to elevated levels of insulin-like growth factors. J Cell Biol 110:1307-1317.

Danielson N, Pettmann B, Vahlsing HL, Manthorpe M, Varon S (1988) Fibroblast growth factor effects on peripheral nerve regeneration in a silicon chamber model. J Neurosci Res 20:320-330.

Denburg JL (1988) Cell-cell recognition in the regenerating neuromuscular system of the cockroach. Am Zool 28:1135-1144.

Devor M, Schneider G (1975) Neuroantomical plasticity: the principle of conservation of total axonal arborization. In: Aspects of neural plasticity (Vital-Durand F, Jeannerod M, eds), pp 191-200. Paris: Editions INSERM.

Engel J, Ganel A, Melamed R, Rimon S, Farine I (1980) Choline acetyltransferase for differentiation between human motor and sensory nerve fibers. Ann Plast Surg 4:376-380.

Esslen E (1960) Electromyographic findings on two types of misdirection of regenerating axons. Electroencephalogr Clin Neurophysiol 12:738-741.

Evans DH, Murray JG (1956) A study of regeneration in a motor nerve with a unimodal fiber diameter distribution. Anat $\operatorname{Rec} 126$ : 311-329.

Goldberg DJ, Schacher S (1987) Differential growth of the branches of a regenerating bifurcate axon is associated with differential axonal transport of organelles. Dev Biol 124:35-40.

Gordon T, Gillespie J, Orozco R, Davis L (1991) Axotomy-induced changes in rabbit hindlimb nerves and the effects of chronic electrical stimulation. J Neurosci 11:2157-2169.

Grothe C, Wewetzer K, Lagrange A, Unsicker K (1991) Effects of basic fibroblast growth factor on survival and choline acetyitransferase development of spinal cord neurons. Dev Brain Res 62:257-261.

Gruber H, Zenker W (1973) Acetylcholinesterase: histochemical differentiation between motor and sensory fibers. Brain Res 51:207214

Hansson HA, Dahlin LB, Danielsen N, Fryklund L, Nachemson AK Polleryd P, Rozell B, Skottner A, Stemme S, Lundborg G (1986) Evidence indicating trophic importance of IGF-I in regencrating peripheral nerves. Acta Physiol Scand 126:609-614.

Innocenti GM (1981) Growth and reshaping of axons in the establishment of visual callosal connections. Science 212:824-827.

Ishii DN (1989) Relationship of insulin-like growth factor II gene expression in muscle to synaptogenesis. Proc Natl Acad Sci USA 86: 2898-2902.

Ivy GO, Killackey HP (1982) Ontogenetic changes in the projections of neocortical neurons. J Neurosci 2:735-743.

Jenq C-B, Coggeshall RE (1984) Regeneration of axons in tributary nerves. Brain Res 310:107-121.
Kanda K, Sato H, Hashizume K, Yamada J (1989) The effects of blocking nerve conduction on retrograde HRP labeling of rat motoneurons. Neurosci Lett 99:153-156.

Kilvington B (1941) Some experiments on nerve regeneration. Aust NZ J Surg 10:266-272.

Kunemund V, Jungalwala FB, Fischer G, Chou DK, Keilhauer G, Schachner M (1988) The L2/HNK-1 carbohydrate of neural cell adhesion molecules is involved in cell interactions. J Cell Biol 106: 213-223.

Langley JN, Anderson HK (1904) The union of different kinds of nerve fibers. J Physiol (Lond) 31:365-391.

Langley JN, Hashimoto $M$ (1917) On the suture of separate nerve bundles in a nerve trunk and on internal nerve plexuses. J Physiol (Lond) 51:318-345.

Liu X-L, Zhu J-K (1990) The histochemical study of rat sciatic nerve cholinesterase in degeneration and regeneration. Reconstr Microsc 6:43-47.

Mackinnon S, Dellon L, O'Brien J (1991) Changes in nerve fiber numbers distal to a nerve repair in the rat sciatic nerve model. Muscle Nerve 14:1116-1122

Maki Y (1991) Experimental study of selective motor and sensory nerve regeneration, Pt 2. J Jpn Soc Surg Hand 7:975-978.

Manthorpe M, Skaper SD, Williams LR, Varon S (1986) Purification of adult rat sciatic nerve ciliary neuronotrophic factor. Brain Res 367 : 282-286.

Martini R, Bollensen E, Schachner M (1988) Immunocytological localization of the major peripheral nervous system glycoprotein Po and the $\mathrm{L} 2 / \mathrm{HNK}-\mathrm{I}$ and $\mathrm{L} 3$ carbohydrate structures in developing and adult mouse sciatic nerve. Dev Biol 129:330-338.

Martini K, Xin Y, Schmitz B, Schachner M (1992) The L2/HNK-I carbohydrate epitope is involved in the preferential outgrowth of motor neurons on ventral roots and motor nerves. Eur J Neurosci 4:628-639.

McManaman JL, Crawford FG, Stewart SS, Appel SH (1988) Purification of a skeletal muscle polypeptide which stimulates choline acetyltransferase activity in cultured spinal cord neurons. J Biol Chem 263:5890-5897.

McManaman JL, Crawford FG, Clark R, Richker J, Fuller F (1989) Multiple neurotrophic factors from skeletal muscie: demonstration of effects of basic fibroblast growth factor and comparisons with the $22 \mathrm{kDa}$ choline acetyltransferase development factor. J Neurochem 53:1763-1771.

McManaman JL, Haverkamp LJ, Oppenheim RW (1991) Skeletal muscle proteins rescue motor neurons from cell death in vivo. Adv Neurol 56:81-88.

Mesulam M-M (1982) Tracing neural connections with horseradish peroxidase. New York: Wiley.

Miledi R, Slater CR (1970) On the degeneration of rat neuromuscular junctions after nerve section. J Physiol (Lond) 207:507-528.

Morris JH, Hudson AR, Weddell G (1972) A study of degeneration and regeneration in the divided rat sciatic nerve based on electron microscopy II. The development of the 'regenerating unit.' Z Zellforsch Mikrosk Anat 124:103-130.

Murray M, Edwards MA (1982) A quantitative study of the reinnervation of the goldfish optic tectum following optic nerve crush. J Comp Neurol 209:363-373.

Ochi M, Fukuhara K, Chow SP, So K-F (1990) Experimental study on selective sensory and motor nerve regeneration using the femoral nerve of the rats. J Jpn Soc Surg Hand 7:45-49.

Oppenheim RW, Prevette D, Qin-Wei Y, Collins F, MacDonald J (1991) Control of embryonic motoneuron survival in vivo by ciliary neurotrophic factor. Science 251:1616-1618.

Pcinado A, Zipser B, Macagno ER (1987a) Regeneration of afferent axons into discrete tracts within peripheral nerves in the leech. Brain Res 410:330-334.

Peinado A, Macagno ER, Zipser B (1987b) A group of related surface glycoproteins distinguish sets and subsets of sensory afferents in the leech nervous system. Brain Res 410:335-339.

Pestronk A, Drachman D (1978) A new stain for quantitative measurement of sprouting at neuromuscular junctions. Muscle Nerve 1:70-74.

Ramon y Cajal S (1928) Degeneration and regeneration of the nervous system. London: Oxford UP.

Redfern PA (1970) Neuromuscular transmission in newborn rats. J Physiol (Lond) 209:701-709.

Riley DA, Sanger JR, Matloub HS, Yousif NJ, Bain JL, Moore GH 
(1988) Identifying motor and sensory myelinated axons in rabbit peripheral nerves by histochemical staining for carbonic anhydrase and cholinesterase activities. Brain Res 453:79-88.

Sanders FK, Young JZ (1946) The influence of peripheral connexion on the diameter of regenerating nerve fibers. J Exp Biol 22:203-212.

Sato H, Kanda K, Hashizume K, Yamada J (1989) Nerve stimulation does not necessarily enhance retrograde HRP labeling of rat motoneuron. Neurosci Lett 107:81-84.

Schachner M (1990) Functional implications of glial cell recognition molecules. Semin Neurosci 2:497-507.

Schmued LC, Fallon J (1986) Fluoro-gold: a new fluorescent retrograde axonal tracer with numerous unique properties. Brain Res 377:147154.

Sendtner M, Kreutzberg GW, Thoenen H (1990) Ciliary neurotrophic factor prevents the degeneration of motor neurons after axotomy. Nature 345:440-441.

Shawe GDH (1955) On the number of branches formed by regenerating nerve-fibers. Br J Surg 42:474-488.

Smalheiser NR, Crain SM (1984) The possible role of "sibling neurite bias" in the coordination of neurite extension, branching, and survival. J Neurobiol 15:517-529.

Stanfield BB, O'Leary DM (1985) The transient corticospinal projection from the occipital cortex during the postnatal development of the rat. J Comp Neurol 238:236-248.

Stelzner DJ, Strauss JA (1986) A quantitative analysis of frog optic nerve regeneration: is retrograde ganglion cell death or collateral axonal loss related to selective reinnervation? J Comp Neurol 245:83106.

Thomas RW, Davenport HA (1949) Axon branching in nerve regeneration and its trophic effect on muscle. Q Bull Northw Univ Med Sch 23:170-176.

Watrous WG (1940) Axon branching after nerve regeneration. Proc Soc Exp Biol Med 44:541-542.

Weiss P, Edds MV (1945) Sensory-motor nerve crosses in the rat. J Neurophysiol 30:173-193.

Zalewski AA (1970) Effects of reinnervation on denervated skeletal muscle by axons of motor, sensory, and sympathetic neurons. Am J Physiol 219:1675-1679. 